\title{
A Distributed Coordination Protocol for the Connectivity Maintenance in a Network of Mobile Units
}

\author{
Tullio Facchinetti \\ University of Pavia \\ tullio.facchinetti@unipv.it
}

\author{
Gianluca Franchino \\ University of Pavia \\ gianluca.franchino@unipv.it
}

\author{
Giorgio Buttazzo \\ Scuola Superiore S. Anna \\ g.buttazzo@sssup.it
}

\begin{abstract}
The coordination of a team made by a set of mobile robots involves several challenges since, given a task to be accomplished by the team, the mobility of each robotic unit is often constrained by several factors. Connectivity is one of the most important factors. Cooperating robots must keep connected for many reasons: to plan a working strategy, to coordinate movements and actions, to re-configure the network in case of unexpected events.

This paper describes a fully distributed coordination strategy expressly targeted to the maintenance of the connectivity among mobile robots. The proposed approach is based on the periodic broadcast of state information, which is used to drive the mobility strategies of nodes. The team uses a Leader/Follower organization, where Leaders have to move toward predefined locations to perform the given task, while Followers have to coordinate to maintain the global network connectivity, i.e., the multi-hop connection between Leaders. The proposed scheme is analyzed to derive its formal properties, and simulation results are presented to show the protocol behaviour under different working conditions.
\end{abstract}

\section{Introduction}

Multi-robots systems have been shown to be one of the most effective solutions for many problems where human direct intervention is dangerous or impossible and single robots can not achieve the desired goal or the desired level of service [6].

As a sample scenario we consider an environmental monitoring application, where a team of heterogeneous robots is deployed in the area under control, where each robot is equipped with specific sensory and actuation devices. The robots interact by exchanging messages on a wireless communication channel. We assume that only a few units can perform a specific action in a given location, so that such units must move to the required location. When the target location is located outside the maximum transmission range of common wireless transceivers, the communication among mobile units can only be achieved through multi-hop message forwarding. This situation is typical in unstructured or hostile environments where no long-range communication technologies can be used (i.e., satellites). In these situations, node trajectories must be carefully planned to maintain the network connectivity at every instant to avoid network partitions. Another example is attested by the interest of DARPA in applications where network connectivity is established and maintained using mobile coordinated robots [1].

The communication among multi-robots represents a common application of Mobile Ad Hoc Networks (MANETs), due to the intrinsic ad hoc nature of a multirobot system [10]. Topology control, which is a key issue in the research field of MANETs [9], is typically based on two techniques: hierarchical topology organization and power control. The former technique, also referred as clustering, aims at organizing the nodes into inter-communicating clusters, each one controlled by a special node, the clusterhead [2]; the challenge is to partition the network to optimize the energy spent by each node to communicate and to have an acceptable robustness (redundancy) of multi-hop communication paths. The latter method aims at calibrating the node transmission power in order to obtain a network with a minimum level of connectivity, while minimizing energy consumption [4].

The techniques described above consider the node mobility in the sense that they can reconfigure some network parameters after an arbitrary motion of one or more nodes. The protocols dynamically adapt the parameters under control to cope with new network topologies. However, they do not act on the robot mobility to achieve the system goal.

The approach presented in this paper explicitly exploits the mobility of robotic units to guarantee a minimum level of network connectivity, where robots trajectories can be planned to avoid network partitions. The nodes move according to a mobility strategy that depends on the state of neighbours. Each node periodically broadcasts state information and its neighbours update their local view of the network to adapt their trajectory. The overall result is the maintenance of the global connectivity to prevent partitions in the network.

Depending on the application, the nodes may have dif- 
ferent capabilities in terms of sensory and actuation skills. For this reason, nodes are divided into Leaders and Followers. Leaders are dedicated to the execution of a specific application task, and are selected depending on their sensory and actuation capabilities. The remaining units, the Followers, are mainly dedicated to the connectivity maintenance.

The proposed technique is fully distributed, in the sense that each robot uses only the information coming from its neighbours to plan its motion. Under the considered mobility model, some interesting formal properties arise from the distributed behaviour of the system.

The paper is organized as follows: Section 2 presents the model for the multi-robot system. Section 3 introduces the classification of nodes. The protocol is described in Section 4, while Section 5 illustrates the mobility strategies applied to different nodes. Related theoretical results are presented in Section 6, while Section 7 shows the results from simulations. Finally, Section 8 concludes the paper.

\section{System model}

The considered systems consists of a set (or team) of $n$ mobile robots $r_{i}, i=1 \ldots n$, also referred as nodes or units. Each node $r_{i}$ is considered as a point characterized by its position $x_{i}$ (considered as a vector). Each node also have an instantaneous speed and acceleration, but since those kinematic parameters are not relevant for the protocol, they have no associated symbols. All nodes are location-aware, meaning that they know their own position at every instant.

This paper considers the following kinematic constraints on the robot mobility:

- the moduli of speed and acceleration are bounded by an upper threshold;

- robots can change direction instantaneously, i.e., no non-holonomic constraints are considered [5].

The nodes communicate by transmitting messages over a wireless medium. The communication model considers a constant communication radius $r$, which is assumed to be the same for all nodes. When node $r_{i}$ transmits a message, a node $r_{j}$ receives the message if and only if $\left\|x_{i}-x_{j}\right\| \leq r$, i.e., its distance from $r_{i}$ is less than or equal to $r$.

The network topology defined by the wireless links among nodes is represented by a graph. Each node is associated with a vertex of the graph. An undirected graph $\mathcal{G}$ consists of a vertex set $\mathcal{V}(\mathcal{G})=\left\{r_{i}: 1, \ldots, n\right\}$ and an edge set $\xi(\mathcal{G}) \subseteq\left\{\left(r_{i}, r_{j}\right): r_{i}, r_{j} \in \mathcal{V}(\mathcal{G})\right\}$ such that, for $i, j=1, \ldots, n,\left(r_{i}, r_{j}\right) \in \xi(\mathcal{G}) \Longleftrightarrow\left(r_{j}, r_{i}\right) \in$ $\xi(\mathcal{G})$. A path in a directed graph $\mathcal{G}$ is a finite sequence $r_{i_{1}}, \ldots, r_{i_{j}}$ of vertices such that $r_{i_{k}}, r_{i_{k+1}} \in \mathcal{V}(\mathcal{G})$ for $k=1, \ldots, j-1$.

Nodes are divided into two categories: Leaders and Followers. A Leader is a node which has been selected to perform a specific action in a given location. The goal of a Leader is to move towards the assigned location, while maintaining a communication link with at least another node. A Follower is a team node devoted to the connectivity maintenance. In other words, its mobility strategy is designed to guarantee the full connectivity of the team.

The results described in this paper consider the case of two Leaders only. It is worth noticing that, while considering only two Leaders may appear as a simplistic scenario, it applies to many interesting practical situations. For example, one of the two Leaders may be a base station and the second Leader may be a mobile unit which need to keep the connectivity with the base station through a multi-hop sequence of intermediate nodes dedicated to the connectivity maintenance. Moreover, the scenario can be easily adapted for using multiple Leaders by keeping independent paths between opportunely chosen pairs of Leaders.

The proposed technique is based on the use of a special path between Leaders, which is formally defined as follows:

Definition 1 Given a graph $\mathcal{G}$ describing the network communication topology, $\mathcal{P}$ is the path made by modes $r_{i_{1}}, r_{i_{2}}, \ldots, r_{i_{m}}$ where

1. $r_{i_{1}}$ and $r_{i_{m}}$ are the Leaders;

2. $\forall r, s: r_{i_{r}} \neq r_{i_{s}}$

Condition 2 means that each vertex belonging to $\mathcal{P}$ is visited once. Moreover, it is worth noticing that may be $m \leq n$, meaning that not all nodes are included into $\mathcal{P}$ at a given instant. On the other hand, $\mathcal{P}$ is meant to dynamically change during the system lifetime, so that is should be written as a function of time, i.e. $\mathcal{P}(t)$. However, the time dependency will be omitted where not explicitly required.

From the connectivity point of view, $\mathcal{P}$ represents the "high priority" path between the two Leaders. In other words, it is the path between the two Leaders which must be always guaranteed by the Followers in order to achieve the full connectivity of the team.

Finally, a working space without obstacles is assumed. The adaptation of the proposed approach to the presence of obstacles is planned as a future work.

\section{Nodes classification}

Considering the path $\mathcal{P}$ introduced in Section 2, the optimal condition is when all nodes are included into $\mathcal{P}$, since it allows the two Leaders to reach the farthest possible locations, i.e. when all Followers lie on the straight line connecting the two Leaders, and each Follower is at the maximum possible distance from its neighbours. However, given a generic network topology, it may not be possible to find such a path. Looking at the Graph Theory, a concept which is close to $\mathcal{P}$ is the Hamiltonian path [7]. The Hamiltonian path is defined as a path that contains all the vertices of a graph, being each node visited exactly 


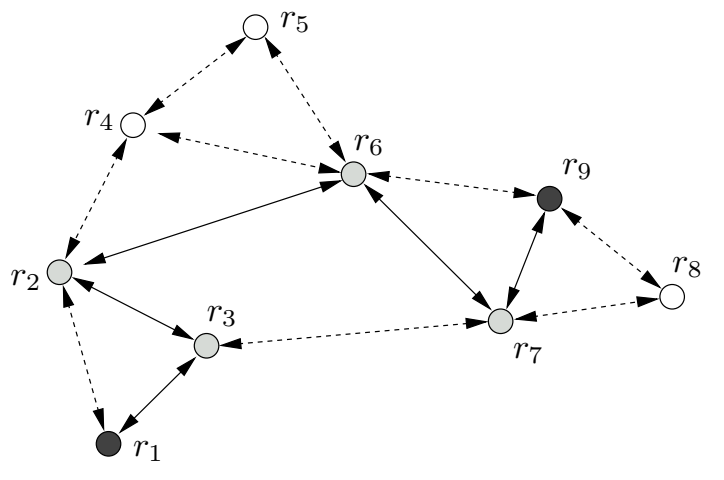

Figure 1. Example of network configuration.

once. For a given graph, an Hamiltonian path has 2 relevant properties in the scope of this paper:

1. it may not exist;

2. the algorithm required to build the path is NPcomplete.

Given the definition of $\mathcal{P}$, it can be noticed that, in the optimal case where $m=n, \mathcal{P}$ is an Hamiltonian path. However, it could be impossible to find such a path for a generic network topology. Therefore, Followers can be classified in two sub-set:

- Followers $r_{i} \in \mathcal{P}$, called Inner Followers (IFs);

- Followers $r_{j} \notin \mathcal{P}$, called Outer Followers (OFs).

Figure 1 shows an example of network configuration where different types of nodes are represented:

- dark gray nodes $r_{1}$ and $r_{9}$ are the Leaders;

- light gray nodes $\left\{r_{2}, r_{3}, r_{6}, r_{7}\right\} \in \mathcal{P}$ are IFs;

- white nodes $\left\{r_{4}, r_{5}, r_{8}\right\} \notin \mathcal{P}$ are OF;

- dotted arrows represent links between nodes which are able to listen to each others;

- solid line arrows represent links between IFs and between Leaders and IFs (nodes included into $\mathcal{P}$ ); notice that such links imply the ability to listen to each others.

$\mathcal{P}$ is Hamiltonian when all Followers are IFs. This condition allows the Leaders to reach the farthest positions between themselves, since the Followers can be arranged on the straight line joining the two Leaders. This configuration achieves the global network connectivity while allowing the highest possible mobility freedom for the Leaders.

$\mathcal{P}$ is a dynamic structure which evolves during the system lifetime. Intuitively, the goal of the mobility strategy of an $\mathrm{OF}$ is to get closer to some IF and thus to be dynamically included into $\mathcal{P}$, thus becoming an IF itself. When all OFs have been included into $P$, the highest possible distance between the two Leaders be safely achieved without loss of connectivity.

It is worth observing that $\mathcal{P}$ is used for maintaining the network connectivity. In case there exists shorter paths between two nodes that need to exchange information, the shortest path is used for the multi-hop message forwarding. For example, given the configuration depicted in Figure 1, the multi-hop communication between $r_{1}$ and $r_{9}$ can be done exploiting the path through $r_{3}$ and $r_{7}$.

\section{Protocol description}

The initial network topology is assumed to be fully connected, i.e., the problem of partitioned networks is not addressed. If network partitions exist, only the connected nodes are able to cooperate. On the other hand, the proposed technique can be independently applied to each subnetwork.

At system startup, there are no differences among nodes in terms of being Leaders, IFs or OFs. The two Leaders are selected among all nodes based on the specific task to be carried out.

Once the Leaders have been selected, the nodes interact to define $\mathcal{P}$, and thus to divide the nodes into IFs and OFs, as described in Section 3. Notice that the proposed protocol is not intended for providing a novel approach to find a path between two nodes within a network. For this purpose, existing techniques can be used. For example, nodes may use a classic routing protocol, like the well known AODV protocol [8], to establish a route between the two Leaders and thus identifying the active neighbours, which become IFs. During the startup, there is no need to find the longest possible path, since $\mathcal{P}$ will be dynamically extended during the system lifetime. Moreover, given an initial network topology, it may be computationally impractical or even impossible to find the path where all nodes are included (an Hamiltonian path). Therefore, the startup procedure is based on the choice of a convenient initial path $\mathcal{P}$, no matter how many nodes are initially included. Then, the protocol leverages the node mobility to dynamically insert all the OFs into $\mathcal{P}$.

After that, each node $r_{i}$ starts to periodically broadcast state information, using the received state information to plan its movements. Mobility strategies used by the nodes are describes in detail in the next section.

\section{Mobility strategy}

Leaders, IFs and OFs have different mobility strategies, due to the different tasks that they must attain. Since an ordering over $\mathcal{P}$ is required to illustrate the protocol behaviour, we introduce the following Definition 2:

Definition 2 Being one of the two Leaders identified with $r_{l}$, the notation $r_{l_{i}}$ indicates the IF which is $i-1$ links far from $r_{l}$ when traversing only links along $\mathcal{P}\left(r_{l}=r_{l_{1}}\right)$.

Notice that the results described in this paper are independent from the Leader considered for labelling the IFs.

\subsection{Mobility of Leaders}

The goal of a Leader is to move toward its target destination to complete the assigned mission. While Leaders 
should typically move as fast as possible to achieve the best system performance, they must explicitly take care to not lose the connection with their closest IF. In other words, using the notation of Definition 2, Leader $r_{l_{1}}$ and $r_{l_{m}}$ must control their speed to keep the connection with $r_{l_{2}}$ and $r_{l_{m-1}}$, respectively.

\subsection{Mobility of Inner Followers}

IFs are team's nodes that form $\mathcal{P}$. The characteristic of IFs is to have at least two IFs as their neighbours.

The goal of $r_{l_{i}}$ is to keep the connection with its two neighbours belonging to $\mathcal{P}$, namely $r_{l_{i-1}}$ and $r_{l_{i+1}}$, also called critical nodes. Therefore, the mobility strategy of $r_{l_{i}}$ only considers the information received from its critical nodes. Notice that, for this purpose, Leaders are considered as IFs: for example, the critical nodes of $r_{l_{2}}$ are $r_{l_{1}}$, which is a Leader, and $r_{l_{3}}$.

The mobility rule adopted for the IF $r_{l_{i}}$ considers the position information received by its critical nodes $r_{l_{i-1}}$ and $r_{l_{i+1}}$, respectively $x_{l_{i-1}}$ and $x_{l_{i+1}}$. Node $r_{l_{i}}$ calculates the medium point $x_{\text {med }}$ as in Equation 1

$$
x_{\text {med }}=\frac{\left(x_{l_{i-1}}+x_{l_{i+1}}\right)}{2} .
$$

$x_{\text {med }}$ represents the target location of $r_{l_{i}}$, which adapts its direction and speed to reach $x_{\text {med }} . x_{\text {med }}$ is updated every time $r_{l_{i}}$ receives newer information from its critical nodes.

\subsection{Mobility of Outer Followers}

The goal of an OF is to get as close as possible to an IF in order to become an IF itself. When an OF is included into $\mathcal{P}$, it is said to be promoted ${ }^{1}$.

To describe the mobility strategy of OFs, the concept of distance of a node from $\mathcal{P}$ is introduced with Definition 3.

Definition 3 A node $r_{i}$ is said to be at distance $g_{i}$ from $\mathcal{P}$ if the shortest path between $r_{i}$ and an arbitrary IF is made by $g_{i}$ links. By default, $g_{i}=0$ if $r_{i}$ is either an IF or a Leader.

Every time a node $r_{i}$ broadcasts information about its own position, it also sends the value of $g_{i}$, letting the neighbours to update their local distance information on the basis of $g_{i}$.

When an OF $r_{j}$ receives the information from a neighbour $r_{i}$, it updates the value of $g_{j}$ using the following rule:

$$
\text { if } g_{j}>g_{i} \text { then } g_{j}=g_{i}+1
$$

In this way, every node $r_{j}$ keeps track of its distance from $\mathcal{P}$ using the information received from its neighbours. Node $r_{j}$ also keeps track of the position $x_{\text {low }}$ of the node $r_{\text {low }}$ that sent the lowest $g_{i}$ value, and updates its mobility strategy to get as close as possible to $r_{\text {low }}$, i.e.

\footnotetext{
${ }^{1}$ The term is taken from the chess terminology. It describes the transformation of a pawn that reaches the eighth rank into a player's chosen piece.
}

driving toward $x_{\text {low }}$. In this way, every OF will follow a node which is closer to $\mathcal{P}$ than itself, thus bringing itself closer and closer to $\mathcal{P}$.

At the startup, after the generation of $\mathcal{P}$, which splits the nodes into Leader, IFs and OFs, the distance values are set so that

$$
g_{i}= \begin{cases}0, & \text { if } i: r_{i} \in \mathcal{P} \\ \infty, & \text { if } i: r_{i} \notin \mathcal{P}\end{cases}
$$

When the OF is sufficiently close to $\mathcal{P}$ (see Section 5.4), it will be promoted. Figure 2 shows an example of network with the indication of distances of OFs from $\mathcal{P}$. The mobility strategy of OFs depicted in Figure 2 makes $r_{6}$ and $r_{7}$ to follow node $r_{5}$, while node $r_{8}$ moves toward $r_{7}$.

\subsection{Promotion of Outer Followers}

The promotion process takes place when a node $r_{j} \notin \mathcal{P}$ (an OF) becomes sufficiently close to an IF. Using the notation of Definition 2, Definition 4 states the formal definition of proximity between $\mathrm{OFs}$ and $\mathcal{P}$.

Definition 4 A node $r_{j} \notin \mathcal{P}$ is said to be sufficiently close to $\mathcal{P}$ when

$$
\exists r_{l_{i}} \in \mathcal{P}:\left\|x_{j}-x_{l_{i}}\right\| \leq r \wedge\left\|x_{j}-x_{s}\right\| \leq r
$$

where $x_{s}$ represents the co-ordinate of node $r_{s} \in \mathcal{P}$, such that may be either $x_{s}=x_{l_{i-1}}$ or $x_{s}=x_{l_{i+1}}$.

Definition 4 states that $r_{j}$ is sufficiently close to $\mathcal{P}$ when $r_{j}$ is able to establish a communication with $r_{l_{i}}$ and at least one of the two neighbours $r_{l_{i-1}}, r_{l_{i+1}} \in \mathcal{P}$ of $r_{l_{i}}$. It is straightforward to prove that this condition can always be achieved, since $r_{j}$ can get arbitrarily close to $r_{l_{i}}$ and the communication radius $r$ is the same for all nodes. When $r_{j}$ is sufficiently close to $r_{l_{i}}, r_{j}$ can be promoted and is therefore inserted into $\mathcal{P}$ between $r_{l_{i}}$ and $r_{s}$.

Figure 2 shows a network configuration where an $\mathrm{OF}$ is sufficiently close to $\mathcal{P}$ to be promoted. The $\mathrm{OF} r_{5}$, which was following node $r_{3} \in \mathcal{P}$, can be inserted into $\mathcal{P}$, since it is close enough to $r_{3}$ to be able to establish a link with $r_{2}$. When this condition is detected by $r_{3}$ and $r_{2}$, the local information of such nodes are updated to insert $r_{5}$ between the two nodes. Therefore, $\mathcal{P}$ becomes $r_{1} \leftrightarrow r_{2} \leftrightarrow r_{5} \leftrightarrow$ $r_{3} \leftrightarrow r_{4}$

\section{Theoretical results}

The theoretical results proved in this section show that, under the considered mobility model, the loss of packets (due to noise and interference) does not affect the correct behaviour of Inner Followers, i.e., the network connectivity achieved by $\mathrm{P}$ is not affected by packet loss. This result derives from the system ability of correctly working independently from the frequency at which the nodes broadcast their state information. This result is stated by Theorem 1 . 


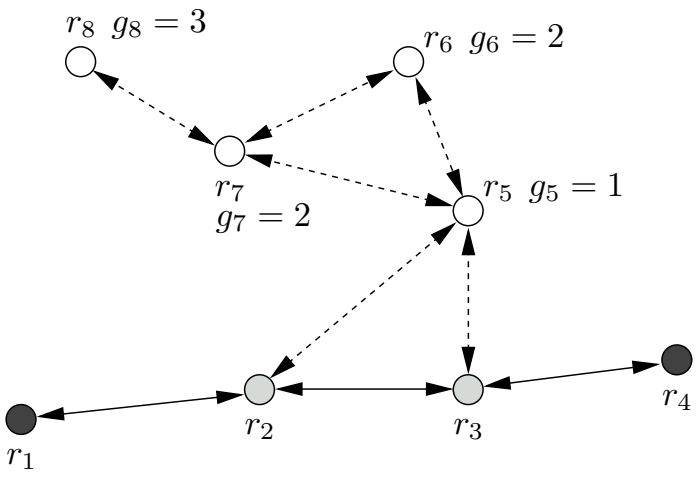

Figure 2. Example of network configuration which allows $r_{5}$ to be added to $\mathcal{P}$.

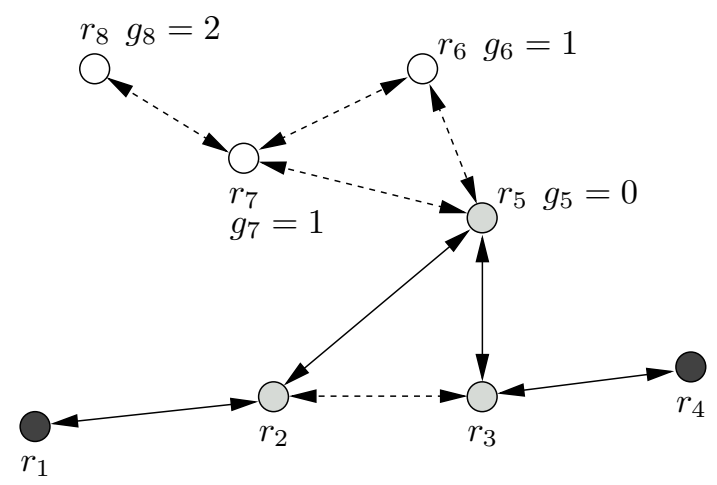

Figure 3. Example of network configuration where $r_{5}$ has been added to $\mathcal{P}$.

Theorem 1 The $\mathcal{P}$ path is never broken during the motion between two consecutive system configurations.

\section{Proof.}

Let us consider 4 nodes $r_{l_{i-1}}, r_{l_{i}}, r_{l_{i+1}}, r_{l_{i+2}} \in$ $\mathcal{P}$. Nodes positions at time $t_{a}$ are respectively $x_{l_{i-1}}, x_{l_{i}}, x_{l_{i+1}}, x_{l_{i+2}}$, and are used by the nodes to calculate their target set-points.

Let $t_{b}>t_{a}$ be the next considered time instant. The notation $x_{i}^{\prime}$ indicates the set-point position of node $r_{i}$ at time $t_{b}$.

From the mobility rule of Inner Followers stated by Equation 1, it holds that

$$
x_{l_{i+1}}^{\prime}=\frac{x_{l_{i+2}}+x_{l_{i}}}{2}
$$

and

$$
x_{l_{i}}^{\prime}=\frac{x_{l_{i+1}}+x_{l_{i-1}}}{2}
$$

Each node, steady or already moving, can turn instantaneously to drive toward the set-point along the straight line connecting the actual location and the set-point.

Subtracting Equation 4 from Equation 3:

$$
x_{l_{i+1}}^{\prime}-x_{l_{i}}^{\prime}=\frac{x_{l_{i+2}}-x_{l_{i}}}{2}-\frac{x_{l_{i+1}}-x_{l_{i-1}}}{2}
$$

which can be rewritten as

$$
2\left(x_{l_{i+1}}^{\prime}-x_{l_{i}}^{\prime}\right)=x_{l_{i+2}}-x_{l_{i+1}}+x_{l_{i}}-x_{l_{i-1}}
$$

The proof continues by proving that $\left\|x_{l_{i+1}}^{\prime}-x_{l_{i}}^{\prime}\right\| \leq r$, being $t_{a}$ and $t_{b}$ arbitrary chosen. From Equation 5 , it follows that

$$
2\left\|x_{l_{i+1}}^{\prime}-x_{l_{i}}^{\prime}\right\|=\left\|x_{l_{i+2}}-x_{l_{i+1}}+x_{l_{i}}-x_{l_{i-1}}\right\|
$$

and applying the triangular inequality to Equation 6

$\left\|x_{l_{i+2}}-x_{l_{i+1}}+x_{l_{i}}-x_{l_{i-1}}\right\| \leq\left\|x_{l_{i+2}}-x_{l_{i+1}}\right\|+\left\|x_{l_{i}}-x_{l_{i-1}}\right\|$

Since the 4 nodes are neighbours into $\mathcal{P}$ and they are linked at time $t_{a}$, this means that

$$
\left\|x_{l_{i+2}}-x_{l_{i+1}}\right\| \leq r
$$

and

$$
\left\|x_{l_{i}}-x_{l_{i-1}}\right\| \leq r
$$

Thus, combining Equation 8 and 9 with Equation 7, it follows that

$$
\left\|x_{l_{i+2}}-x_{l_{i+1}}\right\|+\left\|x_{l_{i}}-x_{l_{i-1}}\right\| \leq 2 r
$$

and, finally, combining Equation 10 with Equation 6, it follows that $2\left\|x_{l_{i+1}}^{\prime}-x_{l_{i}}^{\prime}\right\| \leq 2 r$, therefore, for any final set-point calculated with the mobility rules of IFs, $\mathcal{P}$ is never broken.

It is worth observing that Theorem 1 is based on the assumption that the robot direction can change instantaneously (Section 2), and thus any communication that may occur at any time $t_{c} \in\left[t_{a}, t_{b}\right]$ makes the receiving nodes to recalculate their set-point. Therefore, the same proof can be applied to the interval $\left[t_{c}, t_{d}\right]$, where $t_{d}$ is the time instant at which the robots will arrive at their newer setpoints.

\section{Simulation results}

Since the formal property of $\mathcal{P}$ is proved by Theorem 1, such that it cannot be broken in any condition, even in presence of arbitrary message loss, simulation results focus on giving a feedback about the protocol performance in realistic scenarios where the message forwarding is done by exploiting shorter paths between the two Leaders. For this purpose, the redundancy level $R(n) \in[0,1]$ is introduced:

$$
R(n)=\frac{L-L_{\min }(n)}{L_{\max }(n)-L_{\min }(n)}
$$

where $L$ is the actual number of bidirectional links, $L_{\max }(n)$ is the maximum number of bidirectional links for a given number of nodes $n$; and $L_{\min }(n)$ is the minimum number of bidirectional links which guarantees the full network connectivity. The redundancy level represents a measure of the number of links that can be safely removed from a given topology without affecting the global 


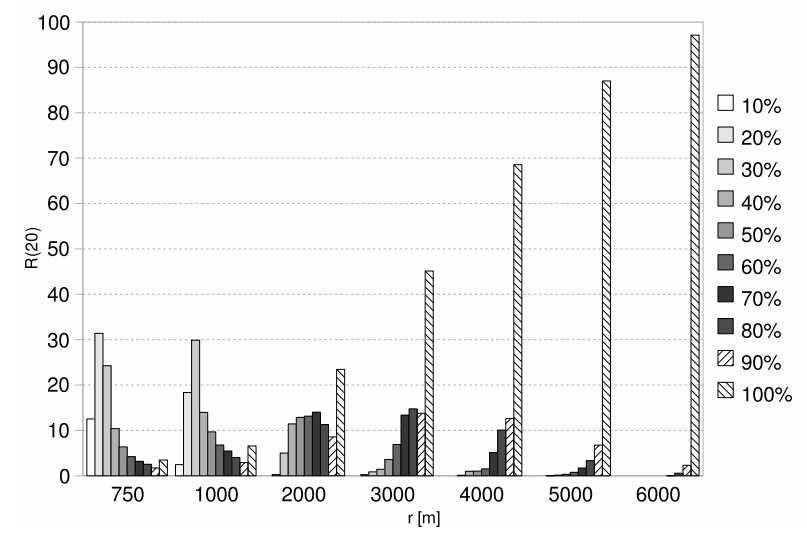

Figure 4. Redundancy level (\%) in a simulation consisting of 20 nodes.

connectivity. It is worth observing that, in the worst case, $\mathcal{P}$ is always available for such a purpose.

Extended simulations have been carried out to assess the redundancy level under different parameter settings. The evaluated parameters are the number of nodes and the transmission radius. For all simulations, the simulation environment is a square with an edge of $10000 \mathrm{~m}$, the virtual tick is $100 \mathrm{~ms}$, the period between two consecutive message broadcasts is randomly chosen in the range $[0.2,5] \mathrm{sec}$, independently for each node, the maximum speed is $10 \mathrm{~m} / \mathrm{s}$, and no packet loss is assumed. The Leaders chose their destination according to the Random Waypoint [3] mobility model, with a constant pause time of $1 \mathrm{sec}$. Finally, for each parameter configuration, a set of $\{3 \sim 5\} \cdot 10^{5}$ simulation runs have been performed, depending on the simulation complexity. Simulation results consist on the distribution of the redundancy level. For each run, at each simulation instant the redundancy level is gathered and quantized in 10 intervals of the range $[0,1]$, and the percentage of simulation time having a given redundancy level is kept. This information can be interpreted in the form "the redundancy level is between $10 \%$ and $20 \%$ for $X \%$ of time". Then, the average on the total number of runs has been calculated for each quantization range.

The derived results show that the variation of $R(n)$ with respect to $n$ is not very significative. Therefore, we report only one result that shows the typical behaviour of the system. Figure 4 shows the redundancy level distribution as a function of $r$ in a simulation consisting of 20 nodes. The minimum value of $r([\mathrm{~m}])$ is chosen so that the two Leaders can reach every pair of locations within the simulated environment. The graph shows $r$ on the $x$ axis, and for each considered radius the $R(n)$ distribution is represented. As $r$ becomes larger, longer time intervals have higher $R(n)$, which is an intuitive result since a larger radius guarantees a larger connectivity area, because less mobility constraints are imposed to the nodes. Moreover, even considering low values of $r, R(n)$ is never null, and thus a minimum redundancy level is achieved in every condition.

\section{Conclusion and future works}

This paper introduced a fully distributed approach to achieve the connectivity maintenance in a team of coordinated mobile robots. The proposed solution is based on a Leader/Follower architecture, where Leaders are nodes that must reach an assigned location, while Followers are dedicated to maintain the connectivity between Leaders. The formal properties of the propose protocol have been shown, as well as simulation results to assess its performance.

Future works include the extension to an unlimited number of leaders, the presence of obstacles, the evaluation of real-time communication constraints and mobile nodes dynamics, and message losses.

Acknowledgment: The authors would like to thank Dr. Gaspare Di Maria for his valuable contribution to the study of the work proposed in this paper.

\section{References}

[1] http://www.darpa.mil/ipto/solicit/open/BAA-0746_PIP.pdf, March 2008.

[2] L. Bao and J. Luna. Topology management in ad hoc networks. In Proceedings of the 4th ACM International Symposium on Mobile Ad Hoc Networking \& Computing (MOBIHOC), pages 129-140, June 2003.

[3] J. Broch, D. A. Maltz, D. B. Johnson, Y.-C. Hu, , and J. Jetcheva. A performance comparison of multi-hop wireless ad hoc network routing protocols. October 1998.

[4] P. Chen, B. O'Dea, and E. Callaway. Energy efficient system design with optimum transmission range for wireless ad hoc networks. In Proceedings of IEEE International Conference on Communications (ICC), volume 2, pages 945-952, April-May 2002.

[5] H. Choset, K. M. Lynch, S. Hutchinson, G. Kantor, W. Burgard, L. E. Kavraki, and S. Thrun. Principles of Robot Motion: Theory, Algorithms, and Implementations. The MIT Press, 2005.

[6] R. Grabowsky, L. E. Navarro-Serment, and C. J. J. Paredis. Heterogeneous teams of modular robots for mapping and exploration. Autonomous Robots, 8(3):293-308, June 2000.

[7] J. Gross and J. Yellen. Graph Theory and its Applications. CRC Press Series on Discrete Mathematics and its Applications, 1999.

[8] C. E. Perkins and E. M. Royer. Ad hoc on-demand distance vector routing. Proceedings of the 2nd IEEE Workshop on Mobile Computing Systems and Applications, pages 90100, February 1999.

[9] G. Srivastava, P. Boustead, and J. F. Chicharo. A comparison of topology control algorithms for ad-hoc networks. In Australian Telecommunications, Networks and Applications Conference (ATNAC), December 2003.

[10] J. Wu and I. Stojmenovic. Ad-Hoc Networks, volume 37. IEEE Computer, 2004. 